TITLE:

\title{
Two-dimensional Raman and infrared vibrational spectroscopy for a harmonic oscillator system nonlinearly coupled with a colored noise bath
}

\author{
$\operatorname{AUTHOR}(\mathrm{S}):$ \\ Kato, T; Tanimura, Y
}

\section{CITATION:}

Kato, T ... [et al]. Two-dimensional Raman and infrared vibrational spectroscopy for a harmonic oscillator system nonlinearly coupled with a colored noise bath. JOURNAL OF CHEMICAL PHYSICS 2004, 120(1): 260-271

\section{ISSUE DATE:}

2004-01-01

URL:

http://hdl.handle.net/2433/49972

\section{RIGHT:}

Copyright 2004 American Institute of Physics. This article may be downloaded for personal use only. Any other use requires prior permission of the author and the American Institute of Physics. 


\title{
Two-dimensional Raman and infrared vibrational spectroscopy for a harmonic oscillator system nonlinearly coupled with a colored noise bath
}

\author{
Tsuyoshi Kato ${ }^{\text {a) }}$ and Yoshitaka Tanimura ${ }^{\text {b) }}$ \\ Institute for Molecular Science, Myodaiji, Okazaki, Aichi 444-8585, Japan
}

(Received 27 August 2002; accepted 2 October 2003)

\begin{abstract}
Multidimensional vibrational response functions of a harmonic oscillator are reconsidered by assuming nonlinear system-bath couplings. In addition to a standard linear-linear (LL) systembath interaction, we consider a square-linear (SL) interaction. The LL interaction causes the vibrational energy relaxation, while the SL interaction is mainly responsible for the vibrational phase relaxation. The dynamics of the relevant system are investigated by the numerical integration of the Gaussian-Markovian Fokker-Planck equation under the condition of strong couplings with a colored noise bath, where the conventional perturbative approach cannot be applied. The response functions for the fifth-order nonresonant Raman and the third-order infrared (or equivalently the second-order infrared and the seventh-order nonresonant Raman) spectra are calculated under the various combinations of the LL and the SL coupling strengths. Calculated two-dimensional response functions demonstrate that those spectroscopic techniques are very sensitive to the mechanism of the system-bath couplings and the correlation time of the bath fluctuation. We discuss the primary optical transition pathways involved to elucidate the corresponding spectroscopic features and to relate them to the microscopic sources of the vibrational nonlinearity induced by the system-bath interactions. Optical pathways for the fifth-order Raman spectroscopies from an "anisotropic" medium were newly found in this study, which were not predicted by the weak system-bath coupling theory or the standard Brownian harmonic oscillator model. (C) 2004 American Institute of Physics. [DOI: 10.1063/1.1629272]
\end{abstract}

\section{INTRODUCTION}

The multidimensional vibrational spectroscopy is the Raman or the infrared analog of the multidimensional nuclear magnetic resonance techniques, and has recently been intensively developed to explore liquid dynamics, ${ }^{1}$ intra- and/or intermolecular vibrational couplings, ${ }^{2-7}$ molecular structure changes, ${ }^{8-12}$ and vibrational wave packet motion in condensed phases. ${ }^{13}$

For the fifth-order Raman spectroscopy, ${ }^{1}$ signals corresponding to various Raman polarizability tensor elements were measured for the intermolecular vibrational modes of liquids $\mathrm{CS}_{2}$ (Refs. 14-16) and solution of $\mathrm{CS}_{2}$ (Ref. 17) by minimizing the cascade contributions, ${ }^{18,19}$ which were underestimated in the initial attempts of experiments. ${ }^{20-22}$ Molecular dynamics simulation techniques were developed ${ }^{23-27}$ and compared with experimental results. ${ }^{28-30}$ For example, the nodal lines of signals observed by Kaufman et al. ${ }^{16}$ were also found in the molecular dynamics simulation studied by Saito and Ohmine, ${ }^{30}$ and were recognized to arise from couplings among intermolecular rotational modes. Theoretical studies for different models probe sensitivities of the multidimensional spectroscopy. ${ }^{31-40}$ For the third-order IR experiments, the femtosecond phase-controlled IR pulses are now

\footnotetext{
${ }^{a)}$ Present address: Department of Chemistry, Graduate School of Science, Tohoku University, Sendai 980-8578, Japan.

${ }^{b}$ Author to whom all correspondence should be addressed. Present address: Department of Chemistry, Graduate School of Science, Kyoto University, Kyoto 606-8502. Electronic mail: tanimura@kuchem.kyoto-u.ac.jp
}

available to obtain the heterodyne detected signal fields from the matter. ${ }^{12}$ The two-dimensional (2D) Fourier plots of the three-pulse vibrational echo technique applied to a dipeptide molecule implies the coupling between two amide-I modes. ${ }^{41}$ The degree of the correlation among vibrational modes in a small molecule and the conformational fluctuation of an $\alpha$-helical peptide are also investigated by the $2 \mathrm{D}$ IR spectroscopy. ${ }^{10,42-46}$

All the sensitivities of the multidimensional vibrational spectroscopy rely upon the multiple pumping and the probing processes which are expressed by the multi-time correlation functions of the polarizability or the dipole moment as a function of the relevant vibrational coordinates. We can utilize there the multiple-resonances, constructive or destructive interferences among the optical pathways beyond onedimensional spectroscopy, i.e., the third-order nonresonant Raman or the first-order IR spectroscopy, to select targeting dynamics of interest. ${ }^{47-55}$ It is well understood that a harmonic system does not show any vibrational nonlinearities. ${ }^{1}$ There must be nonlinear sources to endow a harmonic vibrational system with the nonlinear response against an external perturbation, e.g., the anharmonicity of the system potential, the nonlinear coordinate dependence of the polarizability or the dipole of the system and the anharmonic vibrational mode couplings. ${ }^{31}$

In the condensed phase, dissipation induced by inelastic interactions between molecules plays an important role in the dynamics of molecular motion. The normal mode Hamiltonian represented by a sum of a few independent harmonic 
oscillators does not involve such effects. It can be introduced by adding the heat-bath degrees of freedom to couple with the normal coordinates. The Brownian oscillator model (BOM) is one of the most successful quantum descriptions of the dissipative process in the condensed phase such as the vibrational energy relaxation. ${ }^{47,56-60}$ In a standard BOM, the system-bath coupling mechanism is assumed as a sum of the bilinear functions represented by the products of the system coordinate and the bath coordinates. Note that this systembath coupling has nothing to do with the vibrational nonlinearity, since the coupling term can be diagonalized with respect to the vibrational coordinates. The total system is, in principle, described by a normal mode Hamiltonian.

If the system-bath coupling mechanism has a nonlinear or anharmonic property, we can expect that this coupling induces vibrational nonlinear spectroscopic properties of the relevant system, and it can be revealed by the higher-order vibrational spectroscopy. This situation can be viewed as an extension of the anharmonic intramolecular mode coupling ${ }^{31}$ to the system-bath coupling. However, it is not trivial to elucidate the corresponding spectroscopic features and to relate them to the microscopic sources of the nonlinearity, because it cannot be diagonalized. The system-bath coupling induces the vibrational nonlinearity as well as the vibrational dephasing processes.

In one-dimensional spectroscopy, the distinct effects of the nonlinear system-bath coupling mechanisms on the vibrational dephasing processes are hidden by other prominent contributions which can also be observed for the standard BOM as shown in our previous paper. ${ }^{61}$ The pure dephasing contribution can only be seen to broaden the spectral width in addition to the line broadening due to the vibrational energy relaxation. Differences of the dissipative processes may be depicted by multidimensional vibrational spectroscopies, since there the vibrational dynamics correlated by more than two successive pumping processes are measured. We are able to disentangle the several dissipative pathways involved and to investigate the relaxation processes in more detail compared to the one-dimensional spectroscopies. Although multidimensional spectroscopy is a sensitive tool to investigate a mechanism of vibrational dephasing, the effect of a nonlinear (anharmonic) system-bath coupling upon a signal is not trivial as mentioned earlier; one has to clarify the features of the response functions governed by a different dephasing mechanism before analyzing a real experimental signal where many other effects may be involved. For this purpose, here we employ a single mode system to analyze the multidimensional spectra induced by the nonlinear system-bath couplings in various conditions.

We consider a model system to describe a vibrational motion in the condensed phase, which is represented by a Hamiltonian defined by

$$
\hat{H}=\hat{H}_{s}+\sum_{j}\left[\frac{1}{2 m_{j}} \hat{p}_{j}^{2}+\frac{1}{2} m_{j} \omega_{j}^{2}\left\{\hat{x}_{j}-\frac{F_{j}(\hat{q})}{m_{j} \omega_{j}^{2}}\right\}^{2}\right],
$$

where

$$
\hat{H}_{s}=\frac{1}{2 M} \hat{p}^{2}+U(\hat{q})
$$

is the Hamiltonian for the relevant optically active oscillator in which $\hat{q}, \hat{p}, M$, and $U(\hat{q})$ denote the coordinate (displacement from the potential minimum), momentum, mass, and the potential. An ensemble of optically inactive harmonic oscillators is assumed as a heat-bath, and the coordinate, momentum, mass, and frequency of the $j$ th bath oscillator are given by $\hat{q}_{j}, \hat{p}_{j}, m_{j}$, and $\omega_{j}$, respectively.

The standard BOM assumes a linear-linear (LL) system-bath coupling with respect to the vibrational coordinates as mentioned before, $\hat{H}_{\mathrm{SB}}=-\Sigma_{j} F_{j}(\hat{q}) \hat{x}_{j}$, where $F_{j}(\hat{q})=c_{j} \hat{q}$ with the coupling constant $c_{j}$. For a harmonic potential, within the weak system-bath coupling, the LL interaction with a white noise reduces the level-dependent population relaxation rate, but is insufficient to induce the pure dephasing, i.e., lifetime limited dephasing. The loss of phase coherence can be achieved by anharmonicities in the system potential, the bath potential, or both, or by a nonlinear system-bath coupling mechanism considered in the present paper. ${ }^{61-64}$

In the context of the BOM, Okumura and Tanimura introduced an interaction term composed of a squared system coordinate and a linear bath coordinate (SL interaction), i.e., $F_{j}(\hat{q})=g_{j} \hat{q}^{2} / 2$ with the coupling constant $g_{j}$, to discuss the effects of pure dephasing on the one-dimensional spectroscopies. ${ }^{65}$ From the viewpoint of the normal mode picture, the SL interaction corresponds to the anharmonic mode coupling. For a harmonic oscillator system with a fundamental frequency $\omega_{0}$, this interaction causes frequency modulations of the system given by $\delta \omega(t)$ $=\sum_{j} g_{j} x_{j}(t) /\left(2 M \omega_{0}\right)$, where $x_{j}(t)$ denotes the classical motion of the bath coordinate in the weak coupling limit. In Ref. 65 , they derived a perturbative expression for a two-time correlation function of the system coordinate (corresponds to spontaneous Raman spectroscopy) and demonstrated that the LL and the SL models are distinguishable by their temperature dependencies. In a real experiment, however, molecular structures and configurations may also be changed by a temperature, it is not so easy to distinguish such effects. In order to verify a mechanism, one need to develop a measurement which is more sensitive to a difference of a dephasing mechanism. Fifth-order Raman and third-order IR spectroscopy are such examples [see Eqs. (6) and (7)]. ${ }^{1,55}$

For the SL model, Steffen and Tanimura calculated one-, two-, and three-time correlation functions of the Raman polarizability or the dipole moment for various coupling strengths and noise correlation times. ${ }^{66-68}$ They derived the quantum Fokker-Planck equation for the SL coupling by generalizing the reduced equation of motion for a GaussianMarkovian (GM) bath ${ }^{69-71}$ to overcome the complexity of the analytical perturbative treatments. The nonlinearity of the system-bath interaction yields an interesting feature in the fifth-order Raman and the third-order IR photon echo response: 2D signal is shown to be useful to measure the degree of the frequency fluctuations (i.e., inhomogeneity) of the vibrational mode. The key to their studies is the inclusion of a noise correlation. In liquids or glasses where the motions of the bath molecules have the same time constant as the motions of the system, the white noise approximation can break down and a more elaborate model is necessary, which 


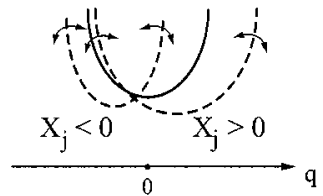

$\mathrm{C} 1 / \mathrm{C}_{2}>0$

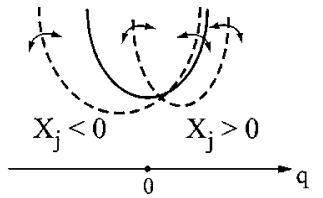

$\mathrm{C}_{1} / \mathrm{C}_{2}<0$
FIG. 1. Schematic illustration of the effects of the system-bath coupling on a relevant harmonic potential system. For simplicity, only the effects of the coupling with the $j$ th bath motion is drawn. The LL coupling $\left(-2 g_{j} x_{j} C_{1} q\right)$ and the SL coupling $\left(-g_{j} x_{j} C_{2} q^{2}\right)$ induce the displacement and the frequency fluctuation of the relevant potential, respectively, and are responsible for $T_{1}$-type and $T_{2}$-type vibrational relaxation processes in the weak coupling limit. The bold lines represent the unperturbed potential, while the dotted-dashed and dashed lines represent the perturbed potential for $C_{2} / C_{1}>0$ (left) and $C_{2} / C_{1}<0$ (right), respectively. These two patterns of the potential distortion reflect the difference of the relative phase between the LL and the SL coupling mechanisms.

accounts for the finite correlation between the system and the bath motions. The simplest example is a GM modulation defined by the noise correlation function $f(t)=\Delta^{2} \exp$ $(-\gamma t),{ }^{69}$ where $\Delta$ and $\gamma$ denote the strength and the correlation of the modulation, respectively. The pure dephasing is then analogous to the $T_{2}^{*}$ process in the nuclear magnetic relaxation, but it cannot be characterized by a single decay constant $1 / T_{2}^{*}$ because of the finite correlation time of the modulation.

In this paper we investigate the effects of the simultaneous presence of the LL and the SL couplings on the vibrational multidimensional spectroscopies in contrast to the above-mentioned studies, where the additivity of the dissipative processes induced by the two coupling mechanisms are implicitly assumed. As shown in our previous study, ${ }^{61}$ it is reasonable to assume the additivity of the dissipative effects for the one-dimensional vibrational spectroscopy. Such an assumption is not valid for the higher-order spectroscopies as shown in this study. Theoretical models for the dissipative processes beyond the simple $T_{1}$ and $T_{2}$ picture, which is based on the weak system-bath coupling and assumes the additivity, can really be pursued through the careful examinations of the multidimensional response functions.

In this study, the system-bath coupling function is represented by

$$
F_{j}(\hat{q})=\frac{g_{j}}{2}\left(2 C_{1} \hat{q}+C_{2} \hat{q}^{2}\right),
$$

where $g_{j}$ is the coupling strength. Constants $C_{1} \geqslant 0$ and $C_{2}$ specify the relative importance of the couplings via the $\hat{q}(\mathrm{LL})$ or $\hat{q}^{2}(\mathrm{SL})$ term, respectively. In Fig. 1 we show the schematic illustration for the LL and the SL coupling mechanisms for a harmonic potential system considered. The LL coupling and the SL coupling induce the displacement and the frequency fluctuation of the relevant potential, respectively, and are responsible for $T_{1}$-type and $T_{2}$-type vibrational relaxation processes in the weak coupling limit. We chose $C_{2}=0$ or \pm 1 to set the relative phase between the two coupling mechanisms [see Sec. III B].

The organization of this paper is as follows: In Sec. II, we summarize the theoretical description of the multidimensional vibrational spectroscopy by a numerical Fokker-
Planck equation approach. In Sec. III A, we estimate of the leading order contributions of the vibrational nonlinear response functions by using the Liouville pathways. The effects of the spatial averaging on the response functions are considered in Sec. III B. Numerical results are presented for the fifth-order Raman and the third-order IR response functions, and are discussed in Sec. IV. The concluding remarks are given in Sec. V.

\section{A FOKKER-PLANCK EQUATION DESCRIPTION OF THE RESPONSE FUNCTIONS}

In this section, we outline the theoretical basis for the calculations of the multidimensional vibrational response functions affected by a GM noise bath. ${ }^{61,66,67,71}$ The vibrational dephasing processes can be determined by the system-bath coupling form [Eq. (3)] and the spectral distribution of the bath oscillators. We assume the bath spectral density expressed as ${ }^{70}$

$$
J(\omega)=\sum_{j} \frac{g_{j}^{2}}{8 m_{j} \omega_{j}} \delta\left(\omega-\omega_{j}\right)=\frac{M \zeta}{\pi} \frac{\omega \gamma^{2}}{\omega^{2}+\gamma^{2}} .
$$

In Eq. (4), a constant $\gamma$ is related to the decay rate of the symmetrized correlation function, $S(t)$, of the collective bath coordinate, $\hat{X} \equiv \sum_{j} g_{j} \hat{x}_{j} / 2$, within the high temperature condition $\beta \hbar \gamma \ll 1\left(\beta=1 / k_{B} T\right.$ with $k_{B}$ and $T$ being the Boltzmann constant and the temperature, respectively);

$$
S(t)=\frac{1}{2}\langle\hat{X}(t) \hat{X}(0)+\hat{X}(0) \hat{X}(t)\rangle_{B}=\frac{M \zeta \gamma}{\beta} e^{-\gamma|t|},
$$

where $\hat{X}(t)$ denotes the Heisenberg representation of $\hat{X}$ and $\langle\cdots\rangle_{B}$ means taking thermal average with respect to the bath degrees of freedom. Therefore, $\tau_{c} \equiv 1 / \gamma$ is a measure of the noise correlation time. $\zeta$ is related to the system-bath coupling strength. The SL coupling strength and the LL coupling strength are defined from Eqs. (1) and (4) by $\zeta_{\mathrm{SL}} \equiv \zeta\left|C_{2}\right|$ and $\zeta_{\mathrm{LL}} \equiv 4\left[C_{1}\right]^{2} \zeta$, respectively. The dimensions of these coupling strength are ${ }^{61}\left[\zeta_{\mathrm{LL}}\right]=\mathrm{s}^{-1}$ and $\left[\zeta_{\mathrm{SL}}\right]=\mathrm{m}^{-2} \mathrm{~s}^{-1}$. A set of four parameters $\zeta_{\mathrm{LL}}, \zeta_{\mathrm{SL}}, \tau_{c}=1 / \gamma$, and $\beta$, thus, completely specifies the system-bath coupling.

The fifth-order Raman and the third-order IR response functions denoted by $R_{\mathrm{Raman}}^{(5)}\left(T_{2}, T_{1}\right)$ and $R_{\mathrm{IR}}^{(3)}\left(T_{3}, T_{2}, T_{1}\right)$, respectively, are defined as the functions of the pulse separation times, $T_{j} \geqslant 0(j=1,2,3)$, as ${ }^{1,48}$

$$
R_{\text {Raman }}^{(5)}\left(T_{2}, T_{1}\right)=\left(\frac{i}{\hbar}\right)^{2}\left\langle\left[\left[\hat{\alpha}\left(T_{1}+T_{2}\right), \hat{\alpha}\left(T_{1}\right)\right], \hat{\alpha}(0)\right]\right\rangle
$$

and

$$
\begin{aligned}
R_{\mathrm{IR}}^{(3)}\left(T_{3}, T_{2}, T_{1}\right)= & \left(\frac{i}{\hbar}\right)^{3}\left\langle\left[\left[\left[\hat{\mu}\left(T_{1}+T_{2}+T_{3}\right), \hat{\mu}\left(T_{1}\right.\right.\right.\right.\right. \\
& \left.\left.\left.\left.\left.+T_{2}\right)\right], \hat{\mu}\left(T_{1}\right)\right], \hat{\mu}(0)\right]\right\rangle,
\end{aligned}
$$

where $\hat{\alpha}(t)=e^{i \hat{H} t / \hbar} \hat{\alpha}(\hat{q}) e^{-i \hat{H} t / \hbar}$ or $\hat{\mu}(t)=e^{i \hat{H} t / \hbar} \hat{\mu}(\hat{q}) e^{-i \hat{H} t / \hbar}$ is the Heisenberg representation of the polarizability or the dipole, and $\langle\cdots\rangle \equiv \operatorname{Tr}\left\{\cdots \hat{\rho}_{\text {eq }}\right\}$, where $\hat{\rho}_{\text {eq }}=e^{-\beta \hat{H}} / \operatorname{Tr}\left\{e^{-\beta \hat{H}}\right\}$ is the thermal equilibrium density operator of the total system. Using the polarizability (dipole) expanded by the coordinate, 
$\alpha(q)=\sum_{n=0} \alpha_{n} q^{n} / n !\left(\mu(q)=\sum_{n=0} \mu_{n} q^{n} / n !\right)$, the response functions are given by a sum of the multi-time correlation functions of the coordinate $\hat{q}$. The second-order IR and the seventh-order Raman response functions, respectively, denoted by $R_{\mathrm{IR}}^{(2)}\left(T_{2}, T_{1}\right)$ and $R_{\mathrm{Raman}}^{(7)}\left(T_{3}, T_{2}, T_{1}\right)$, are obtained by exchanging the polarizability $\alpha(q)$ and the dipole $\mu(q)$ in Eqs. (6) and (7), respectively. In general, the $N$ th-order IR response function is obtained from the $(2 N+1)$ th-order Raman response function by replacing $\alpha(q) \rightarrow \mu(q)$ as suggested in Refs. 1 and 48 (vice versa). In this paper, results are presented only in terms of the fifth-order Raman and the third-order IR response functions for clarity.

Formal expressions of the response functions given by Eqs. (6) and (7) are recast into

$$
\begin{aligned}
R_{\text {Raman }}^{(5)}\left(T_{2}, T_{1}\right)= & \operatorname{Tr}\left\{\alpha(\hat{q}) e^{-i \mathcal{L} T_{2} / \hbar} \frac{i}{\hbar} \alpha^{\times}(\hat{q})\right. \\
& \left.\times e^{-i \mathcal{L} T_{1} / \hbar} \frac{i}{\hbar} \alpha^{\times}(\hat{q}) \hat{\rho}_{\mathrm{eq}}\right\}
\end{aligned}
$$

and

$$
\begin{aligned}
R_{\mathrm{IR}}^{(3)}\left(T_{3}, T_{2}, T_{1}\right)= & \operatorname{Tr}\left\{\mu(\hat{q}) e^{-i \mathcal{L} T_{3} / \hbar} \frac{i}{\hbar} \mu^{\times}(\hat{q})\right. \\
& \times e^{-i \mathcal{L} T_{2} / \hbar} \frac{i}{\hbar} \mu^{\times}(\hat{q}) \\
& \left.\times e^{-i \mathcal{L} T_{1} / \hbar} \frac{i}{\hbar} \mu^{\times}(\hat{q}) \hat{\rho}_{\mathrm{eq}}\right\},
\end{aligned}
$$

respectively, where $X^{\times} \ldots=[\hat{X}, \ldots]$ for $\hat{X}=\hat{\alpha}$ or $\hat{\mu}$, and $\mathcal{L} \ldots=[\hat{H}, \ldots]$ is the Liouvillian. ${ }^{72}$ These expressions provide us more intuitive pictures on the response functions. For example, the right-hand side of Eq. (8) can be read from right to left; the thermal equilibrium state is modified by the first interaction with the impulsive laser pulses via the polarizability at $t=0$, then it evolves in time for $T_{1}$ governed by the Liouvillian, and modified again by the second interaction with the electric field at $t=T_{1}$ followed by the time propagation for $T_{2}$. Then the state of the system is probed at $t$ $=T_{1}+T_{2}$ through its Raman polarization.

Because the laser field is assumed to only interact with the system via the polarizability (dipole), the reduced description of the optical processes can be made. The reduced density operator of the relevant system is obtained by tracing out the optically inactive bath degrees of freedom from the density operator of the total system. ${ }^{47,73,74}$ We made the reduced description of the system dynamics by using the Wigner distribution function defined by ${ }^{75,76}$

$$
W_{0}(p, q, t) \equiv \frac{1}{2 \pi \hbar} \int_{-\infty}^{\infty} d r e^{i p r / \hbar} \rho\left(q-\frac{r}{2}, q+\frac{r}{2}, t\right),
$$

where $\rho\left(q, q^{\prime}, t\right)$ is the reduced density matrix element in the coordinate representation. The Wigner function represents the system dynamics as an evolution of the probability distribution in the phase space, $(p, q)$. The quantum FokkerPlanck equation, which governs the time evolution of the Wigner function, can be cast into a hierarchical form: ${ }^{61,66,67,71}$

$$
\frac{\partial}{\partial t} W_{0}(p, q, t)=-\mathcal{L}_{s} W_{0}(p, q, t)-\Phi_{W}(p, q) W_{1}(p, q, t),
$$

$$
\begin{aligned}
\frac{\partial}{\partial t} W_{1}(p, q, t)= & -\left\{\mathcal{L}_{s}+\gamma\right\} W_{1}(p, q, t) \\
& -\Phi_{W}(p, q) W_{2}(p, q, t) \\
& +\Theta_{W}(p, q) W_{0}(p, q, t),
\end{aligned}
$$

$\vdots$

$\frac{\partial}{\partial t} W_{n}(p, q, t)=-\left\{\mathcal{L}_{s}+n \gamma\right\} W_{n}(p, q, t)$

$$
\begin{aligned}
& -\Phi_{W}(p, q) W_{n+1}(p, q, t) \\
& +n \Theta_{W}(p, q) W_{n-1}(p, q, t),
\end{aligned}
$$

and

$$
\begin{aligned}
\frac{\partial}{\partial t} W_{N}(p, q, t)= & -\left\{\mathcal{L}_{s}+N \gamma\right\} W_{N}(p, q, t) \\
& +\Gamma_{W}(p, q) W_{N}(p, q, t) \\
& +N \Theta_{W}(p, q) W_{N-1}(p, q, t),
\end{aligned}
$$

where $W_{n}(p, q, t)(1 \leqslant n \leqslant N)$, are the auxiliary functions introduced to treat the memory effects of the GM noise, Eq. (5). $\mathcal{L}_{s}$ is the deterministic quantum Liouvillian of the system. ${ }^{77}$ The Fokker-Planck equation in a hierarchical form can handle the GM bath from weak to strong system-bath couplings under the high temperature condition. ${ }^{71,78}$

Bath-induced relaxation operators are given by ${ }^{61}$

$$
\begin{aligned}
\Phi_{W}(p, q)= & -2\left(C_{1}+C_{2} q\right) \frac{\partial}{\partial p}, \\
\Theta_{W}(p, q)= & 2 \zeta \gamma\left\{\left(C_{1}+C_{2} q\right)\left(p+\frac{M}{\beta} \frac{\partial}{\partial p}\right)\right. \\
& \left.+C_{2} \frac{\hbar^{2}}{4} \frac{\partial^{2}}{\partial p \partial q}\right\},
\end{aligned}
$$

and

$$
\begin{aligned}
\Gamma_{W}(p, q)= & 4 \zeta\left(C_{1}+C_{2} q\right)^{2} \frac{\partial}{\partial p}\left(p+\frac{M}{\beta} \frac{\partial}{\partial p}\right) \\
& +\hbar^{2} \zeta C_{2}\left(C_{1}+C_{2} q\right) \frac{\partial^{3}}{\partial p^{2} \partial q} .
\end{aligned}
$$

We note that within the LL model $\left(C_{2} \equiv 0\right)$ the explicit coordinate dependencies of the relaxation operators (12)-(14) vanish. In the limit of the Gaussian-white (GW) bath $\gamma$ $\rightarrow \infty$, i.e., $\quad J(\omega) \rightarrow M \zeta \omega / \pi$ in Eq. (4) and $S(t)$ $\rightarrow 2 M \zeta_{S L} \delta(t) / \beta$ in Eq. (5), the dynamics of the relevant system is described by a single differential equation $[N=0$ in Eq. (11d)], and the single operator of Eq. (14) governs the vibrational relaxation. The Wigner function description of the system is suitable for our numerical investigation. The Wigner function is a real valued function in contrast to the 
(a)

\begin{tabular}{|c|c|c|c|}
\hline 0 & 1 & 0 or 2 & 1 \\
\hline 0 & 0 & 0 & 0 \\
\hline
\end{tabular}

(b)

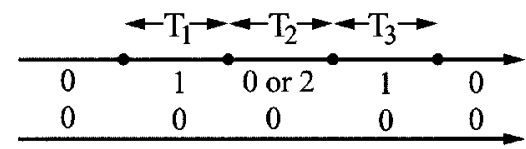

\begin{tabular}{lllll}
\hline 0 & 1 & 1 & 1 & 0 \\
0 & 0 & 1 & 0 & 0 \\
\hline
\end{tabular}

FIG. 2. Examples of the Liouville paths for (a) the fifth-order Raman response and (b) the third-order IR response with a linear polarizability or a dipole $X(\hat{q})=X_{1} \hat{q}$ for $X=\alpha$ or $\mu$ in the weak system-bath coupling limit. Time runs from left to right and the system-laser field interactions $(\propto \hat{q}$ $=\hat{a}+\hat{a}^{\dagger}$ ) are denoted by the dots, where the last(probe) interaction is put on the upper line, and the other dots can be put on both lines. We assume an initial population state $\hat{\rho} \propto \Sigma_{n} \rho_{n, n}|n\rangle\langle n|$. The vibrational quantum number of ket (upper) and bra (lower) sides are depicted for $n=0$. To yield the finite contribution, the final (rightmost) state must be diagonal. (a) There is no Liouville path contributing to the fifth-order Raman response. (b) Eight configurations for the system-laser field interactions are possible for the third-order IR response, but as a whole they do not contribute to the response because of the destructive interferences among them when the dephasing rate is level independent.

complex valued reduced density matrix elements, $\rho\left(q, q^{\prime}, t\right)$, and the expression of the vibrational relaxation operators become much simpler compared with the ones in the energy level representation. ${ }^{61}$ Derivation of the Fokker-Planck equation and the numerical implementation of the time integration are described in detail in the previous paper. ${ }^{61} \mathrm{Cal}-$ culation steps of the fifth-order Raman response function for the GW bath are presented in the Appendix to show how the nonlinear response functions defined in Eqs. (8) and (9) are related to the Wigner function.

\section{PROPERTIES OF THE MULTI-TIME RESPONSE FUNCTIONS}

\section{A. Estimation of leading order of response functions}

To interpret the calculated signals, we will use the Liouville paths associated with the fifth-order Raman and the third-order infrared optical processes. If we assume a relation, $\alpha_{n} \gg \sqrt{M \omega_{0} / \hbar} \alpha_{n+1}$ (or $\mu_{n} \gg \sqrt{M \omega_{0} / \hbar} \mu_{n+1}$ ), and a weak system-bath coupling, the leading order of the response function with respect to $\alpha_{n}$ (or $\mu_{n}$ ) is readily estimated. ${ }^{79}$ We first consider the Liouville paths for the fifth-order Raman response function by explicitly expanding the commutator in Eq. (8) by the polarizability, $\alpha(q)$ $=\sum_{n=0} \alpha_{n} q^{n} / n$ !. If a linear polarizability, $\alpha(q)=\alpha_{1} q$, is assumed, one cannot compose any contributing Liouville paths to the fifth-order response as is explained in Fig. 2(a). With the three(odd)-times of system-laser field interactions one cannot close the diagram with a diagonal element. Note $\hat{q} \propto \hat{a}+\hat{a}^{\dagger}$, where $\hat{a}$ and $\hat{a}^{\dagger}$ denote, respectively, the annihilation and the creation operator for the vibrational state of the system. Thereby, the second lowest order term, $\left[\alpha_{1}\right]^{2} \alpha_{2}$, becomes the leading term. This coincides with the BOM prediction. ${ }^{1}$ Next we consider the third-order IR response. There are eight possible Liouville paths for the system-laser field interactions, Fig. 2(b). It can be shown that these diagrams do not contribute to the response as a whole because of the destructive interference among Liouville paths if the population decay rate and the dephasing rate between any two vibrational levels are both level independent. Such the destructive interference was first pointed out in Ref. 1 for the $\mathrm{BOM}$, and the leading order of $\left[\mu_{1}\right]^{2}\left[\mu_{2}\right]^{2}$ was assumed. In the study of vibrational echo spectroscopy for a harmonic oscillator, Fourkas and co-workers analyzed this cancellation under a weak system-bath coupling condition. They showed that the signal proportional to $\left[\mu_{1}\right]^{4}$ arises if the dephasing rate constants depend on the vibrational states. ${ }^{80}$

To conclude this section, we should point out the validity of a diagrammatic approach. This approach is convenient to analyze the peak positions related to the higher-order vibrational optical transitions, if the system-bath interaction is weak and does not mix the reduced density matrix elements in the course of the time evolution, ${ }^{81}$ e.g., the cases where the vibrational coherence transfer, vibrational population feeding ${ }^{82}$ can be safely neglected. If such mixing processes become significant, many intermediate states are generated from a single vibrational population or the coherence state. Accordingly, many Liouville paths must be taken into account in addition to the ones shown in Fig. 2 for a given configuration of the system-laser field interactions, ${ }^{79,80,82-84}$ which makes the diagrammatic approach practically impossible.

\section{B. Symmetric properties of response functions}

In this section, we will consider the properties of the response functions upon a spatial averaging, although we cannot discuss the detailed molecular reorientational motion as done in Ref. 43. It is not apparent whether the response function will remain finite or not by the spatial averaging, since the polarizability or the dipole, and the Hamiltonian of Eq. (1) do not possess the coordinate inversion symmetry.

We first treat the $(2 N+1)$ th-order Raman response function $R_{\text {Raman }}^{(2 N+1)}$. It is specified by the notation $R_{\operatorname{Raman}( \pm \pm)}^{(2 N+1)}$, where the signs in the subscript specify the parameters for the calculation; the first sign symbolizes the polarizability, $\alpha(q)= \pm \alpha_{1} q+\alpha_{2} q^{2} / 2$, and the second one assigns the relative phase between the LL and the SL coupling constants [sign of $C_{2}$ in Eq. (3)]. The sign of $\alpha_{1}$ represents the orientation of the vibrational coordinate to a laboratory frame. The LL and SL couplings are naturally assumed to have equal weight for both signs after a statistical average as is explicitly demonstrated by the molecular dynamics simulation. ${ }^{85}$ The Hamiltonian of Eq. (1) represents the vibrational motion that has fixed orientation to a laboratory frame, and after the ensemble average it should describe the isotropic property of the system. Response functions satisfy a relation,

$$
R_{\operatorname{Raman}(a, p)}^{(2 N+1)}=R_{\operatorname{Raman}(-a,-p)}^{(2 N+1)}(a, p= \pm),
$$


since the coordinate inversion $q \rightarrow-q$ should not change the value of the response functions. For simplicity, we assume that there are only two relative phases between the LL and the SL couplings, \pm , and define an "isotropic" response function as

$$
\begin{aligned}
R_{\mathrm{Raman}(\mathrm{iso})}^{(2 N+1)}= & \left\{R_{\mathrm{Raman}(++)}^{(2 N+1)}+R_{\mathrm{Raman}(+-)}^{(2 N+1)}+R_{\mathrm{Raman}(--)}^{(2 N+1)}\right. \\
& \left.+R_{\mathrm{Raman}(-+)}^{(2 N+1)}\right\} / 4 \\
= & \left\{R_{\mathrm{Raman}(++)}^{(2 N+1)}+R_{\mathrm{Raman}(-+)}^{(2 N+1)}\right\} / 2,
\end{aligned}
$$

where in the first line we count all the configurations, and the second line is reduced by the coordinate inversion property of Eq. (15). For a linear polarizability $\left(\alpha_{1} \neq 0, \alpha_{2}=0\right)$, a relation $R_{\operatorname{Raman}(+, p)}^{(2 N+1)}=(-1)^{N+1} R_{\operatorname{Raman}(-, p)}^{(2 N+1)}$ holds from Eq. (8), thus we have $R_{\mathrm{Raman}(++)}^{(2 N+1)}=R_{\mathrm{Raman}(--)}^{(2 N+1)}$ $=(-1)^{N+1} R_{\text {Raman (+-) }}^{(2 N+1)}$ and the "isotropic" response functions become

$$
R_{\text {Raman(iso) }}^{(5)}\left(T_{2}, T_{1}\right)=0
$$

and

$$
\begin{aligned}
R_{\text {Raman(iso) }}^{(7)}\left(T_{3}, T_{2}, T_{1}\right) & =R_{\operatorname{Raman}(++)}^{(7)}\left(T_{3}, T_{2}, T_{1}\right) \\
& =R_{\operatorname{Raman}(+-)}^{(7)}\left(T_{3}, T_{2}, T_{1}\right) .
\end{aligned}
$$

The same line of arguments also applies to the $N$ th-order IR response functions $R_{\mathrm{IR}}^{(N)}$ with $\mu(q)= \pm \mu_{1} q+\mu_{2} q^{2} / 2$. We have "isotropic" response functions for the linear dipole $\left(\mu_{1} \neq 0, \mu_{2}=0\right)$,

$$
R_{\mathrm{IR}(\mathrm{iso})}^{(2)}\left(T_{2}, T_{1}\right)=0
$$

and

$$
\begin{aligned}
R_{\mathrm{IR}(\mathrm{iso})}^{(3)}\left(T_{3}, T_{2}, T_{1}\right) & =R_{\mathrm{IR}(++)}^{(3)}\left(T_{3}, T_{2}, T_{1}\right) \\
& =R_{\mathrm{IR}(+-)}^{(3)}\left(T_{3}, T_{2}, T_{1}\right) .
\end{aligned}
$$

Note that Eqs. (17) and (20) are compatible with the leading order terms proportional to $\left[\alpha_{1}\right]^{2} \alpha_{2}$ and $\left[\mu_{1}\right]^{4}$, respectively, mentioned in the preceding section.

In the following considerations, we assume that the laser pulses are impulsive and their envelopes are described by the delta functions, ${ }^{47}$ then the spectroscopic signal is given by the response function itself. For finite pulse envelopes, the signal depends on the experimental layout due to the phasematching condition. ${ }^{86}$

\section{RESULTS AND DISCUSSIONS}

\section{A. Fifth-order Raman (or second-order IR) response functions}

We begin with a comparison of the unaveraged response functions for different system-bath coupling mechanisms in the GW bath $(\gamma \rightarrow \infty)$. Figure 3 shows the contour plots of $R_{\text {Raman(++) }}^{(5)}\left(T_{2}, T_{1}\right)$ at $300 \mathrm{~K}$ as a function of $T_{1}$ and $T_{2}$ for (a) the LL model, (b) and (c) the LL plus SL model, and (d) the SL model. The fundamental energy of the system oscillator $\hbar \omega_{0}$ is $38.7 \mathrm{~cm}^{-1}\left(2 \pi / \omega_{0}=861 \mathrm{fs}\right)$, which is the typical value for the intermolecular oscillation in the condensed phase. The LL coupling strength is taken as $\zeta_{\mathrm{LL}} /\left(\hbar \omega_{0}\right)$

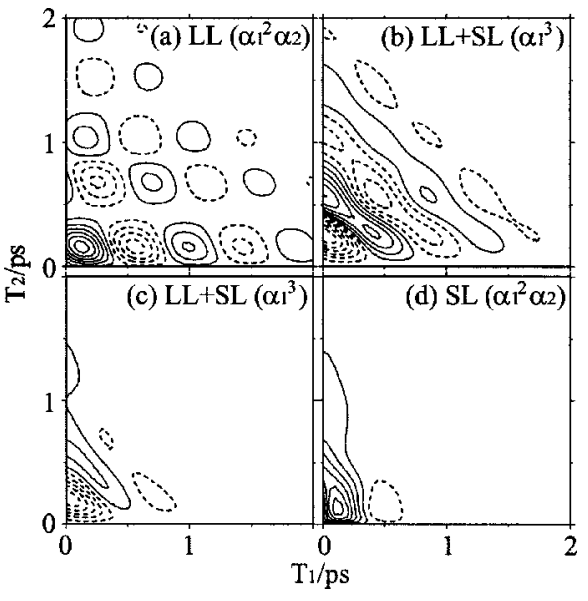

FIG. 3. Contour plots of the fifth-order Raman response functions $R_{\text {Raman(++) }}^{(5)}\left(T_{2}, T_{1}\right)$ for (a) the LL model, (b) and (c) the LL plus SL model, and (d) the SL model at $300 \mathrm{~K}$ in the GW bath. The LL coupling strength is taken $\zeta_{\mathrm{LL}} /\left(\hbar \omega_{0}\right)=0.26$ for $(\mathrm{a})-(\mathrm{c})$, and the dimensionless SL coupling strength $\zeta_{\mathrm{SL}}^{\prime}$ is set to be 0.01 for (b), and 0.1 for (c) and (d). The solid (broken) contours represent positive (negative) values. The leading order of the response function is depicted in each plot (see the text for details).

$=0.26\left[\zeta_{\mathrm{LL}}=10 \mathrm{~cm}^{-1}\right]$ for $(\mathrm{a})-(\mathrm{c})$, and the SL coupling strength $\zeta_{\mathrm{SL}}^{\prime} \equiv \hbar \zeta_{\mathrm{SL}} /\left(M \omega_{0}^{2}\right)$ is set to be 0.01 (weak coupling) for (b) and 0.1 (strong coupling) for (c) and (d). The leading order term of the response function is depicted in each plot, which is determined by obtaining a finite response function starting the numerical calculation with $\alpha(q)=\alpha_{1} q$. When the response function vanishes for the linear polarizability, calculation with $\alpha(q)=\alpha_{1} q+\alpha_{2} q^{2} / 2$ is made. Therefore, the response functions depicted in panels (a) and (d) are calculated with $\alpha(q)=\alpha_{1} q+\alpha_{2} q^{2} / 2$ and the response functions in (b) and (c) are calculated with $\alpha(q)=\alpha_{1} q$ in Fig. 3. A ratio $\widetilde{\alpha}_{2} / \widetilde{\alpha}_{1}=\sqrt{\hbar /\left(M \omega_{0}\right)} \alpha_{2} / \alpha_{1}$ is set to be $10^{-2}$. The response functions for the LL plus SL model, (b) and (d), were not changed by the inclusion of $\alpha_{2}$ term with the present ratio, and $R_{\operatorname{Raman}(+-)}^{(5)}\left(T_{2}, T_{1}\right)$ was identical to $-R_{\text {Raman }(++)}^{(5)}\left(T_{2}, T_{1}\right)$ as indicated in Sec. III B. The response functions for the LL plus SL model show the opposite initial phase compared with the other two models. Response functions for models (b)-(d) depend on the temperature; they get large as the temperature decreases, while the response function for the LL model is temperature independent for our calculations between $T=150$ and $450 \mathrm{~K}$ (not shown). By the differences of the leading order terms and the temperature dependencies, it is clearly indicated that the optical pathways contributing in the LL plus SL model and the other two are different. The response for the LL plus SL model is regarded as the "anisotropic" response $\left(\propto\left[\alpha_{1}\right]^{3}\right)$ because of Eq. (17), whereas those of the LL and the SL models are "isotropic" $\left(\propto\left[\alpha_{1}\right]^{2} \alpha_{2}\right)$ as mentioned in the following.

In Fig. 4, we explain the leading order terms for the LL plus SL model and for the SL model by using the Liouville paths, where the system-bath coupling is considered as a perturbation. Dots represent the system-laser field interactions via the $\alpha_{1}$ term as in Fig. 2, and the vertices denote the system-bath interactions with their explicit functional forms. Initial diagonal vibrational states of the relevant system and 
(a)

\begin{tabular}{|c|c|c|c|c|c|}
\hline & \multicolumn{2}{|c|}{$\mathrm{qx}_{\mathrm{j}}$} & \multicolumn{2}{|c|}{$q^{2} x_{j}$} & \\
\hline 0 & 1 & 0 & 1 & 1 & 0 \\
\hline 0 & 0 & 0 & 0 & 0 & 0 \\
\hline
\end{tabular}

(b)

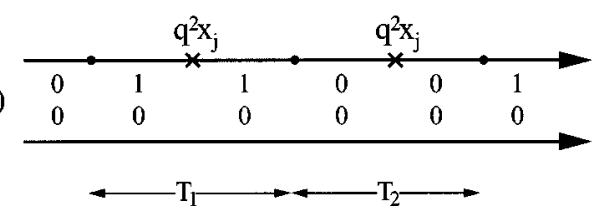

FIG. 4. Examples of the possible Liouville paths for the fifth-order Raman response within the perturbative treatment of the system-bath interactions for (a) finite contribution and (b) no contribution. The vertex denotes a system-bath interaction with the corresponding operator depicted. To leave the bath mode be diagonal, even number of vertices are required for each bath mode. (a) The LL plus SL model; the system and the bath modes can be diagonal at the end. (b) The SL model; even number of system quanta is changed by a system-bath interaction, thus it requires an $\alpha_{2}$ term instead of an $\alpha_{1}$ term. The same is applied for the LL coupling case (not shown).

the bath are assumed. To produce a finite response it is necessary to close the diagram with the diagonal states of the bath modes. This condition is equal to requiring an even number of vertices for each bath mode. Figure 4(a) shows one example of the Liouville path contributing to the response proportional to $\left[\alpha_{1}\right]^{3}$, where the one-quantum coherence state of the system is converted to the population state by a LL interaction with the $j$ th bath mode during the $T_{1}$ evolution, $|1\rangle\langle 0|\rightarrow| 0\rangle\langle 0|$, and the coherence state is preserved through a SL interaction during the $T_{2}$ evolution, $|1\rangle\langle 0|\rightarrow| 1\rangle\langle 0|$. The quantum number of the $j$ th bath mode is conserved after the second SL interaction as $|n\rangle_{j} \rightarrow \mid n$ $\pm 1\rangle_{j} \rightarrow|n\rangle_{j}$ via the succeeding one-phonon emission(absorption) and absorption(emission) processes, where $|n\rangle_{j}$ denotes the energy eigenstate of the $j$ th bath mode. By these system-bath interactions, in total, an odd number of quanta $(=1)$ of the relevant system is changed, and this compensates the odd quantum number change caused by the system-laser field interactions to end up with the diagonal state of the relevant system. On the other hand, within the SL interaction, such compensation does not occur since the quantum number change caused by a single SL interaction must be $\Delta n=0$ or \pm 2 as shown in Fig. 4(b). Therefore, one cannot compose any Liouville paths with a diagonal element of the system at the final time. A finite response is expected beyond the linear polarizability approximation and the contributing terms will be proportional to $\left[\alpha_{1}\right]^{2} \alpha_{2}$ and $\left[\alpha_{2}\right]^{3}$, where the former is the leading order term for the present case. This argument for the SL model is also applied to the LL model, since even times of the LL interactions bring about an even quantum number change in the relevant system. For a response function proportional to $\left[\alpha_{1}\right]^{2} \alpha_{2}$, we note a relation $R_{\operatorname{Raman}(++)}^{(5)}=R_{\operatorname{Raman}(-+)}^{(5)}=R_{\operatorname{Raman}(+-)}^{(5)}$, where we used $\left[-\alpha_{1}\right]^{2} \alpha_{2}=\left[\alpha_{1}\right]^{2} \alpha_{2}$ and Eq. (15). This means that the response functions shown in Figs. 3(a) and 3(d) represent the "isotropic" responses defined by Eq. (16). Thus, we can understand that the simultaneous presence of the LL and the SL coupling mechanisms is crucial for the fifth-order Raman response to be proportional to $\left[\alpha_{1}\right]^{3}$, which is realized by optical pathways different from those found in the theoretical

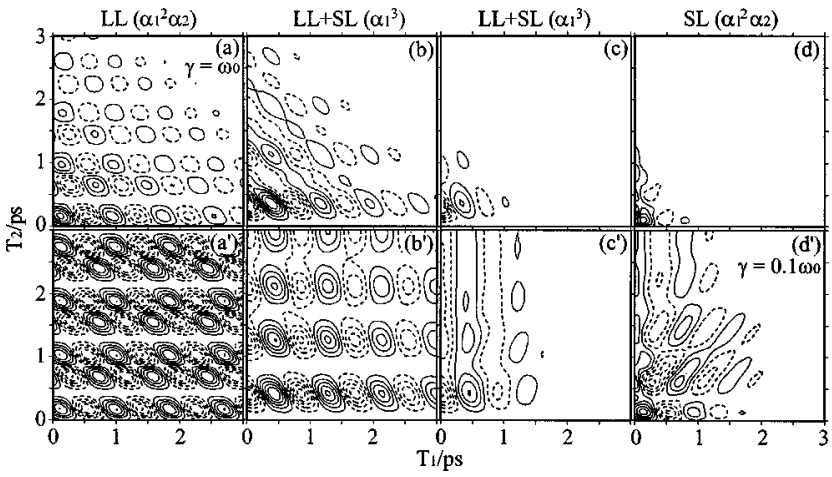

FIG. 5. Effects of the finite correlation time of the GM bath on the fifthorder Raman response functions $R_{\operatorname{Raman}(++)}^{(5)}\left(T_{2}, T_{1}\right)$ for (a) the LL model, (b) and (c) the LL plus SL model, and (d) the SL model at $300 \mathrm{~K}$. The upper panels are for a short correlation time $\gamma=\omega_{0}$ (fast modulation), while the lower panels are for a long correlation time $\gamma=0.1 \omega_{0}$ (slow modulation). The system-bath coupling parameters are the same as used in Fig. 3.

studies so far. ${ }^{1,79}$ We should note, however, that the SL coupling is not the only candidate to lead $\left[\alpha_{1}\right]^{3}$ dependence of the leading order terms. For example, the anharmonicity of the system potential causes such a contribution. ${ }^{72}$ Systembath couplings which do not cause energy loss (quantum transition) in the relevant system, that induce the pure dephasing process, ${ }^{82,87}$ may play the same role as the present SL coupling mechanism does if they operate with the LL coupling mechanism. ${ }^{61,87-90}$

From the comparison of the response function shown in Fig. 3(d) and the analytical result obtained by the stochastic theory, it was suggested that the decay of the signal along the $T_{1}$ and $T_{2}$ axes, respectively, correspond to the pure dephasing and the population decay processes ${ }^{66}$ Here, we compare the response functions shown in Figs. 3(b) and 3(c). We deduce that the decay times along the $T_{1}$ and $T_{2}$ axes in the LL plus SL model are also responsible for the pure dephasing and the population decay time, respectively, although an oscillatory response along the $T_{2}$ axes is observed. We can see a faster decay along the $T_{1}$ axis in Fig. 3(c) (strong SL coupling) compared to Fig. 3(b) (weak coupling) because of the increase of the pure dephasing contribution. ${ }^{61}$ On the other hand, the decay along the $T_{2}$ axis is hardly affected by the change of the SL coupling strength since it mainly reflects the population decay process.

It was demonstrated that the potential fluctuations of the system $^{67,84}$ can be probed as an echo signal in the fifth-order Raman response functions, where the leading order term is proportional to $\left[\alpha_{1}\right]^{2} \alpha_{2}$ and includes the rephasing paths; one-quantum coherence state $|n+1\rangle\langle n|$ during the $T_{1}$ time evolution is inverted to $|n+1\rangle\langle n+2|$ via the second system-field interaction with the $\alpha_{2}$ term for the $T_{2}$ evolution. In Fig. 5 we now illustrate the response functions $R_{\text {Raman }(+,+)}^{(5)}$ in the GM bath with different correlation times $\tau_{c}=1 / \gamma$ for (a) the LL model, (b) and (c) the LL plus SL model, and (d) the SL model at $300 \mathrm{~K}$. The upper panels show the response functions for a short correlation (fast modulation), $\tau_{c}=1 / \omega_{0}$ and the lower panels for a long correlation (slow modulation), $\tau_{c}=10 / \omega_{0}$. In the case of a longer bath correlation time, the system is expected to have a 


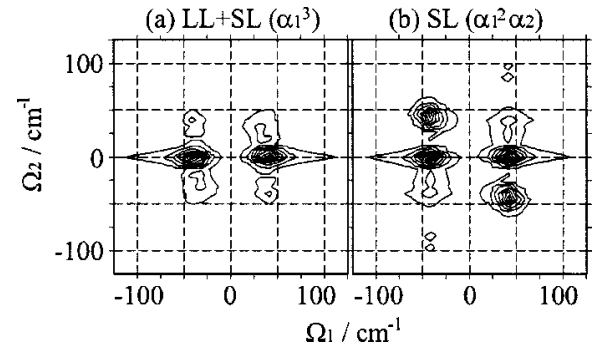

FIG. 6. Plots of the fifth-order Raman response function in the frequency domain, $\left|\widetilde{R}_{\text {Raman }}^{(5)}\left(\Omega_{2}, \Omega_{1}\right)\right|$, for (a) the LL plus SL model, and (b) the SL model. These correspond to Figs. $5\left(\mathrm{c}^{\prime}\right)$ and $5\left(\mathrm{~d}^{\prime}\right)$, respectively. The parameter set is the same as used in Fig. 5.

more inhomogeneous character. In the fast modulation, Figs. $5(a)-5(d)$, response functions are similar to those for the GW bath shown in Fig. 3. On the other hand, in the slow modulation, we observe qualitative changes of the response functions from the GW case, especially in the response functions with the SL coupling. In the LL plus SL models, Figs. 5( $\left.\mathrm{b}^{\prime}\right)$ and $5\left(\mathrm{c}^{\prime}\right)$, we observe a slowly decaying component along the $T_{2}$ axis for $T_{1}$ less than the dephasing time $\leqslant 2$ ps. For the SL coupling, Fig. $5\left(\mathrm{~d}^{\prime}\right)$, the echolike peaks parallel to the $T_{1}=T_{2}$ direction in the time region about $0 \leqslant T_{1}, T_{2} \leqslant 2 \mathrm{ps}$ are clearly observed as well as the slowly decaying component along the $T_{2}$ axis. Note that the effective system-bath coupling strength becomes weaker by the use of the longer correlation time for the present model as mentioned in Ref. 61. The definite absence of the echolike component in the LL plus SL model clearly indicates that the rephasing paths $\left(\propto\left[\alpha_{1}\right]^{2} \alpha_{2}\right)$ are not the primary contributor to the optical response. This fact is evidenced by plotting the response functions in the frequency domain. The spectrum is defined by $\widetilde{R}_{\text {Raman }}^{(5)}\left(\Omega_{2}, \Omega_{1}\right) \equiv \int_{0}^{\infty} d T_{2} \int_{0}^{\infty} d T_{1} e^{i\left(\Omega_{2} T_{2}+\Omega_{1} T_{1}\right)}$ $\times R_{\text {Raman }}^{(5)}\left(T_{2}, T_{1}\right)$. In Fig. 6 , we show the absolute value of $\widetilde{R}_{\text {Raman }}^{(5)}\left(\Omega_{2}, \Omega_{1}\right)$ corresponding to Figs. $5\left(\mathrm{c}^{\prime}\right)$ and $5\left(\mathrm{~d}^{\prime}\right)$, where the echo(rephasing) signal corresponds to the peaks at $\left(\Omega_{1}, \Omega_{2}\right) \sim\left(\mp \omega_{0}, \pm \omega_{0}\right)$. The rephasing peaks seen in the SL model, Fig. 6(b), are not observed in the LL plus SL model, Fig. 6(a), and it implies that the vibrational system with inhomogeneous character does not necessarily induce the echo signal.

Now we move to the analysis of the "isotropic" fifthorder response functions for the LL plus SL model. The response functions for the LL and SL models are "isotropic" as explained for Figs. 3(a) and 3(d), and the "anisotropic" responses are not realized for these models. Figure 7 shows $R_{\text {Raman(iso) }}^{(5)}\left(T_{2}, T_{1}\right)$ for the GM bath; (a) and (b) are the plots of calculated responses with the weak and strong SL coupling constants together with the LL coupling constant in the fast modulation regime, respectively, whereas $\left(a^{\prime}\right)$ and $\left(b^{\prime}\right)$ are for the slow modulation. These results should be compared with the "anisotropic" counterparts shown in Fig. 5 $\left[(b),\left(b^{\prime}\right),(c)\right.$, and $\left.\left(c^{\prime}\right)\right]$. The phases of the response functions are inverted from their "anisotropic" counterparts. This is because the contribution proportional to $\left[\alpha_{1}\right]^{3}$, the main contributor for the "anisotropic" response, is totally cancelled out by the spatial averaging and the second leading order term proportional to $\left[\alpha_{1}\right]^{2} \alpha_{2}$ plays a dominant role. In

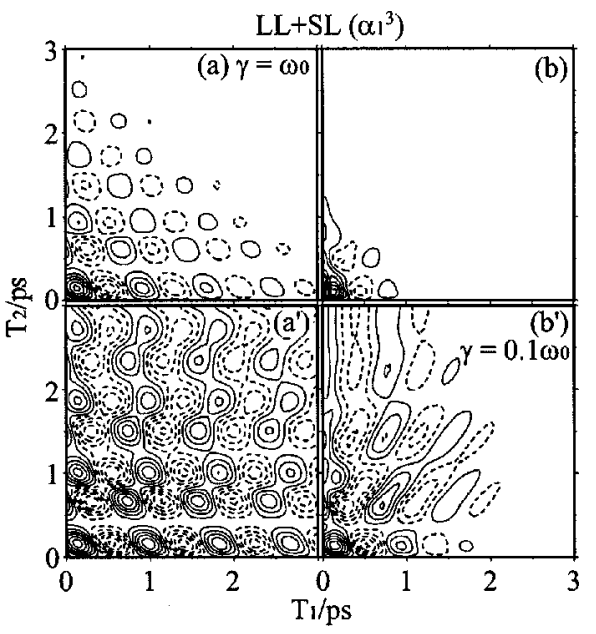

FIG. 7. Plots of the "isotropic" fifth-order Raman response function for the LL plus SL model at $300 \mathrm{~K}$ in the GM bath. The upper panels, (a) and (b), are for the short correlation time $\gamma=\omega_{0}$ (fast modulation), while the lower panels, $\left(a^{\prime}\right)$ and $\left(b^{\prime}\right)$, are for the long correlation time $\gamma=0.1 \omega_{0}$ (slow modulation). The LL coupling strength $\zeta_{\mathrm{LL}} /\left(\hbar \omega_{0}\right)$ is 0.26 and the dimensionless SL coupling strength $\zeta_{\mathrm{SL}}^{\prime}$ is set to be (a) 0.01 and (b) 0.1 . The meaning of contour lines is the same as in Fig. 3. Note that (a) and (b) correspond to the "anisotropic" counterpart shown in Figs. 5(b) and 5(c), respectively.

other words, for the "isotropic" responses the optical pathways remain the same throughout the whole range of the system-bath couplings. Thereby, with a strong SL coupling constant for a GM bath, Fig. 7( $\left.b^{\prime}\right)$, we can clearly see the echolike peaks due to the rephasing paths as opposed to the "anisotropic" response in the LL plus SL model, Fig. 5(c').

\section{B. Third-order IR (or seventh-order Raman) response functions}

We now consider the third-order IR response functions, that have an equivalent form to those of the seventh-order Raman responses. Figure 8 shows the contour plots of the response functions $R_{\mathrm{IR}(++)}^{(3)}\left(T_{3}, 0, T_{1}\right)$ for the GW noise calculated by (a) the LL model, (b) and (c) the LL plus SL model, and (d) the SL model. Each three-dimensional response function is plotted in two dimension as a function of $T_{1}$ and $T_{3}$ by setting the second controllable time $T_{2}$ in Eq. (9) to be zero. In the third-order measurement, all the response functions considered here are regarded as "isotropic." For the contributions proportional to $\left[\mu_{1}\right]^{4}$ or $\left[\mu_{1}\right]^{2}\left[\mu_{2}\right]^{2}$, a relation, $R_{\operatorname{IR}(+,+)}^{(3)}=R_{\operatorname{IR}(-,+)}^{(3)}$, holds, and the coordinate inversion now reads $R_{\mathrm{IR}(-,+)}^{(3)}=R_{\mathrm{IR}(+,-)}^{(3)}$. Thus, we have $R_{\mathrm{IR}(+,+)}^{(3)}=R_{\mathrm{IR}(+,-)}^{(3)} \equiv R_{\mathrm{IR}(\mathrm{iso})}^{(3)}$. Signals in Fig. 8 have the same temperature dependencies as the corresponding response functions explained for the fifth-order Raman response (Fig. 3). The effect of the SL coupling can be observed in the leading order of the response functions. Only the LL model needs $\mu_{2}$ terms, whereas other system-bath coupling models give finite responses only via $\mu_{1}$ terms. This is because, in the LL model, the perfect destructive interference among the possible Liouville paths proportional to $\left[\mu_{1}\right]^{4}$ plays a role. Differences of the leading optical pathway between the LL model $\left(\propto\left[\mu_{1}\right]^{2}\left[\mu_{2}\right]^{2}\right)$ and the other two models $\left(\propto\left[\mu_{1}\right]^{4}\right)$ are seen as the phase differences of 


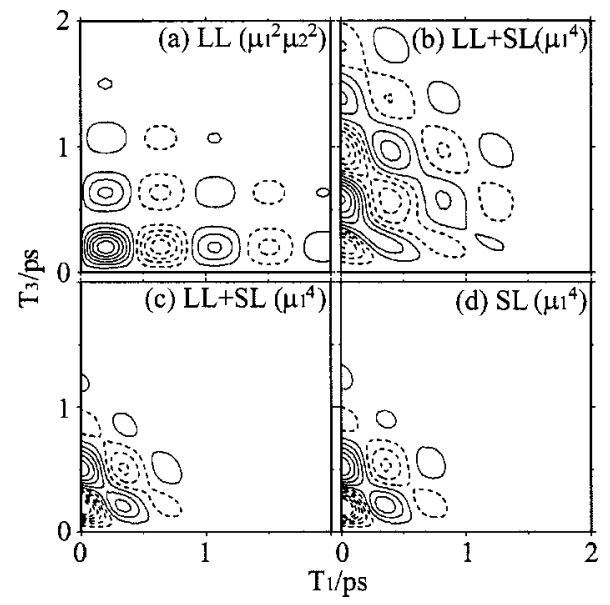

FIG. 8. Plots of the third-order IR response function for (a) the LL model, (b) and (c) the LL plus SL model, and (d) the SL model in the GW bath. The second time variable $T_{2}$ is set to be zero. The parameters and the meaning of the contour lines are the same as used in Fig. 3.

the response functions in Fig. 8. We plot the complemented absolute value of the Fourier spectrum $\widetilde{R}_{\mathrm{IR}}^{(3)}\left(\Omega_{3}, \Omega_{1}\right)$ $\equiv \int_{0}^{\infty} d T_{3} \int_{0}^{\infty} d T_{1} e^{i\left(\Omega_{3} T_{3}+\Omega_{1} T_{1}\right)} R_{\mathrm{IR}}^{(3)}\left(T_{3}, 0, T_{1}\right)$ in Fig. 9. It is clear that the LL plus SL and the SL models, Figs. 9(b)-9(d), exhibit basically the same spectra, where the one-quantum coherences $\left(\Omega_{1}, \Omega_{3} \sim \pm \omega_{0}\right)$ play a dominant role. On the other hand, in Fig. 9(a), the LL model, we can see that the two-quantum coherences and vibrational populations $\left(\Omega_{3}\right.$ $\left.\sim \pm 2 \omega_{0}, 0\right)$ contribute to the response beside the onequantum coherences $\left(\Omega_{1} \sim \pm \omega_{0}\right)$.

The third-order IR or the seventh-order Raman response function has its sensibility to discriminate the SL coupling mechanism from the LL coupling as seen in Figs. 8 and 9. However, the spectrum does not differentiate the LL plus SL model from the SL model, because the primary optical processes are the same $\left(\propto\left[\mu_{1}\right]^{4}\right)$ in these two cases as discussed for the "isotropic" fifth-order Raman responses.

As mentioned in Sec. III A, the finite response with a linear dipole is the manifestation of the incompleteness of the destructive interference among the possible Liouville

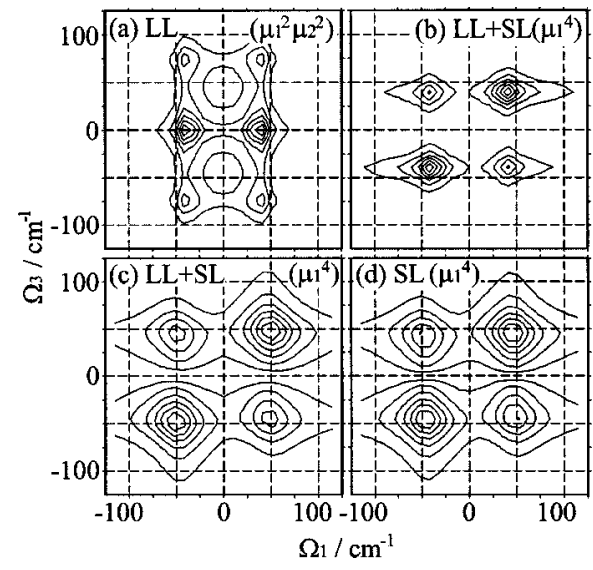

FIG. 9. Plots of the third-order IR spectrum $\left|\widetilde{R}_{\mathrm{IR}}^{(3)}\left(\Omega_{3}, \Omega_{1}\right)\right|$ for (a) the LL model, (b) and (c) the LL plus SL model, and (d) the SL model. The parameters are the same as used in Fig. 3.

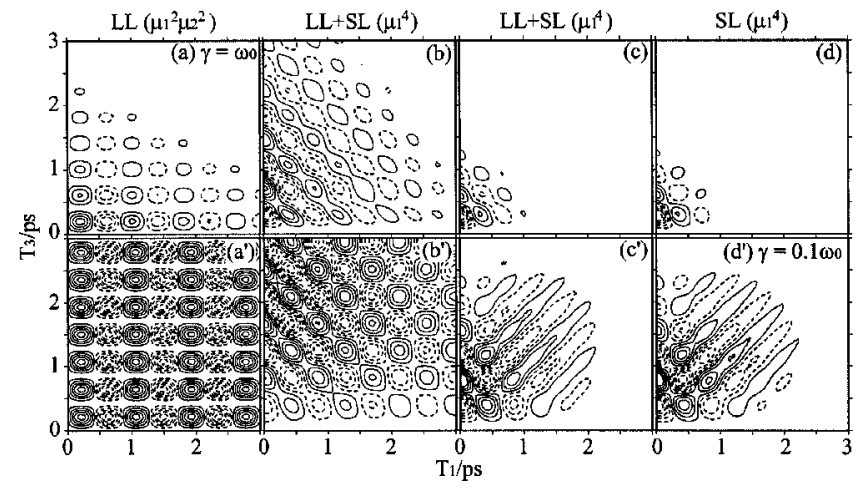

FIG. 10. Effects of the finite correlation time of the GM bath on the thirdorder IR response function for (a) the LL model, (b) and (c) the LL plus SL model, and (d) the SL model. The upper panels are for the short correlation time $\gamma=\omega_{0}$, while the lower panels are for the long correlation time $\gamma$ $=0.1 \omega_{0}$. The parameters and the meaning of the contour lines are the same as used in Fig. 3.

paths. Fourkas et al. calculated the resonant vibrational echo signal under the linear dipole approximation $\left(\propto\left[\mu_{1}\right]^{4}\right)$ with a weak system-bath coupling assumption. Their conclusion suggests that the response is present when the coupling to the bath is linear ( $\sim \mathrm{LL}$ coupling), and is absent when the coupling to the bath is quadratic ( $\sim$ SL coupling) ${ }^{80}$ This statement seems to oppose our results shown in Fig. 8. This is because the explicit form of the system-bath interaction they used was different from ours. Qualitatively, it seems natural to pursue the origin of the nonlinearity for the (LL plus) SL models to the explicit coordinate dependence of the systembath coupling as defined by Eq. (3); the nonlinearity of the system-bath interaction determines the nonlinearity of the system. ${ }^{80}$

The vibrational echo response is expected in the thirdorder IR spectroscopy. ${ }^{12,91-95}$ In Fig. 10, we present $R_{\mathrm{IR}(++)}^{(3)}\left(T_{3}, 0, T_{1}\right)$ with the GM noise for the different bath correlation times with (a) the LL model, (b) and (c) the LL plus SL model, and (d) the SL model at $300 \mathrm{~K}$. The upper panels show the response functions for a short correlation $\tau_{c}=1 / \omega_{0}$, while the lower panels for a long correlation $\tau_{c}$ $=10 / \omega_{0}$, respectively. The system-bath coupling parameters are the same as used in Fig. 5. In the fast modulation, Figs. 10 (a) -10 (d), the responses are similar to those calculated for the GW bath, shown in Fig. 8. We can see that when the SL coupling strength is appreciable and the bath correlation time is long, the echolike signals parallel to $T_{3}=T_{1}$ appear. For the LL plus SL coupling model, the similarity of the profiles in Figs. 8(b) and 8(c) in the GW bath is not preserved in Figs. $10\left(b^{\prime}\right)$ and $10\left(c^{\prime}\right)$ in the GM bath, because the difference of the SL coupling strength is enhanced through the rephasing path contributions.

\section{CONCLUDING REMARKS}

In this paper, we calculated the fifth-order Raman and the third-order IR response functions for a mixed LL and SL system-bath coupling model by using the quantum FokkerPlanck equation. We demonstrated that the whole time profiles of the response functions change dramatically with the form of the system-bath coupling. We found in particular 
that the fifth-order Raman or the second-order IR response function shows "anisotropic" response for the LL plus SL coupling mechanism, where the leading order terms correspond to the optical processes composed of three successive one-quantum optical transitions $\left(\propto\left[\alpha_{1}\right]^{3}\right)$. In contrast, the standard Brownian harmonic oscillator model predicts "isotropic" response which includes a two-quantum optical transition $\left(\propto\left[\alpha_{1}\right]^{2} \alpha_{2}\right)$.

The perturbation theory with respect to the anharmonic potential $V_{\text {anh }}(q)=g_{3} q^{3} / 3 !+g_{4} q^{4} / 4 !+\cdots$ of a harmonic oscillator predicts ${ }^{68}$ that the leading order of the fifth-order Raman response function is $g_{3}\left[\alpha_{1}\right]^{3}$, and that for the thirdorder IR response is $g_{4}\left[\mu_{1}\right]^{4}$, respectively. Therefore, from a viewpoint of the leading order term, the present calculations indicate that the LL plus SL system-bath coupling plays the same role as that of the third-order anharmonic potential $g_{3} q^{3} / 3$ ! for the "anisotropic" fifth-order Raman response. However, for the third-order IR response, the coupling mechanism plays the same role as the fourth-order anharmonicity $g_{4} q^{4} / 4$ ! does. The LL system-bath coupling mechanism can be diagonalized, ${ }^{63}$ therefore, after this diagonalization, the SL coupling is regarded as the third-order anharmonicity of the system potential. For the description of the vibrational echo signal, the third-order anharmonic potential has been pursued as the origin of the vibrational dephasing and the optical nonlinearity. ${ }^{63,96}$ In the previous paper, ${ }^{61}$ on the other hand, we showed that the SL coupling mechanism can partly be recognized to serve as the fourthorder system potential when the noise correlation is long compared with the harmonic frequency (same as speculated by the leading order term of the third-order IR response above). In these respects, the interrelation between the nonlinear system-bath coupling and the anharmonicity of the system potential on the origin of the multidimensional vibrational response functions has not been fully clarified. To elucidate the information that the multidimensional vibrational response function carries, further study is needed from the microscopic viewpoint.

Very recently, Ma and Stratt put forward such a theoretical attempt $\mathrm{t}^{35,36}$ by using the extended instantaneous-normalmode analysis of the molecular dynamics simulation of liquid Xe. They studied the molecular origins accounting for the lack of the echolike signal in the fifth-order Raman response function, and pointed out the relative importance of the dynamical third-order anharmonic potential for each normal mode to understand the fifth-order Raman response. It is interesting to compare Figs. $5\left(\mathrm{c}^{\prime}\right)$ and $5\left(\mathrm{~d}^{\prime}\right)$ with the response functions labeled by "ANH" and "NL" in Fig. 9 of Ref. 35, respectively. Although the response functions are calculated by very different theoretical approaches, the time profiles connected by their leading order terms seems to share the main qualitative feature of the response functions.

Exploring the present study for a higher frequency vibrational mode $\left(\hbar \omega_{0} \gg k_{B} T\right)$ and to extend to a multimodes anharmonic potential system are important to compare them with experimental results and simulations, and to grasp the underlying physical contents of spectroscopy. They can be implemented through an extension of the methods used in this paper, although it requires intensive numerical calcula- tions. We shall address this issue in a future work. In addition to the features observed in this paper, we expect complex time profiles due to the mode couplings and the anharmonicity of the modes, which cannot be resolved by the onedimensional spectroscopy. We are expecting that the present paper and our previous interpretations of the anharmonic coupling effects will be helpful to analyze the experimental results.

\section{ACKNOWLEDGMENTS}

One of the authors (Y.T.) is thankful for financial support from a Grant-in-Aid for Scientific Research (B) (12440171) from Japan Society for the Promotion of Science and Morino Science Foundation.

\section{APPENDIX: CALCULATION STEPS FOR THE FIFTH-ORDER RAMAN RESPONSE FUNCTION FOR THE GAUSSIAN-WHITE BATH}

In this Appendix, we present the calculation steps of the fifth-order Raman response function defined in Eq. (8) to show how the nonlinear response functions are related to the Wigner distribution function. For the GW bath, we only need the Wigner distribution function $W_{0}(p, q, t)$ that is governed by a single Fokker-Planck equation of Eq. (11d) with $N$ $=0$ because of the vanishing correlation time of the bath. The expression in Eq. (8) reads

$$
\begin{aligned}
R_{\operatorname{Raman}}^{(5)}\left(T_{2}, T_{1}\right)= & \operatorname{Tr}\left\{\alpha_{W}^{\mathrm{o}} e^{-\left(\mathcal{L}_{s}-\Gamma_{W}\right) T_{2}}\right. \\
& \left.\times \alpha_{W} e^{-\left(\mathcal{L}_{s}-\Gamma_{W}\right) T_{1}} \alpha_{W} W_{0}^{\mathrm{eq}}\right\},
\end{aligned}
$$

where $W_{0}^{\mathrm{eq}}(p, q)$ denotes the thermal equilibrium Wigner function, and $\alpha_{W}^{\mathrm{o}}$ and $\alpha_{W}$ are the Wigner representations that correspond to the anticommutator, $1 / 2\{\alpha(\hat{q}, \cdots)\}$ and the commutator, $i / \hbar[\alpha(\hat{q}), \cdots]$, respectively. ${ }^{61}$ Tr means the integration over the momentum $p$ and the coordinate $q$. The thermal equilibrium state is calculated by integrating Eq. (11d) from time $t=-t_{i}<0$ to $t=0$ with a trial initial condition,

$$
W_{0}\left(p, q,-t_{i}\right)=\mathcal{N} e^{-\beta\left[p^{2} /(2 M)+U(q)\right]},
$$

where $\mathcal{N}$ is the normalization constant, in which $t_{i}$ was set to be few ps. The time evolution of the Wigner function for $T$ represented by $e^{-\left(\mathcal{L}_{s}-\Gamma_{W}\right) T} W_{0}(t)$ is evaluated by using the second-order Runge-Kutta method applied to the finite difference expression of Eq. (11d). Thereby, we obtain the response function as a function of the two successive time evolution periods of $T_{1}$ and $T_{2}$ by using the corresponding intermediate Wigner functions of

$$
W_{0}\left(T_{1}\right)=e^{-\left(\mathcal{L}_{s}-\Gamma_{W}\right) T_{1}} \alpha_{W} W_{0}^{\mathrm{eq}}
$$

and

$$
W_{0}\left(T_{2}, T_{1}\right)=e^{-\left(\mathcal{L}_{s}-\Gamma_{W}\right) T_{2}} \alpha_{W} W_{0}\left(T_{1}\right),
$$

respectively.

For the GM bath, we implemented the simultaneous time integration of Eq. (11) for the interval $t_{i} \geqslant 1 / \gamma$ to obtain thermal equilibrated auxiliary functions, $W_{n}^{\mathrm{eq}}(p, q)$, with a trial initial condition, $W_{n}\left(p, q,-t_{i}\right)=0(1 \leqslant n \leqslant N)$. The evalua- 
tion of the response function is carried out in the same way as for the GW bath. The depth of the hierarchy $N$ should fulfill $N \gamma>4 \omega_{0}$ and $N \gamma>4 \zeta_{\mathrm{SL}}$ for the present study.

${ }^{1}$ Y. Tanimura and S. Mukamel, J. Chem. Phys. 99, 9496 (1993).

${ }^{2}$ A. Tokmakoff, M. J. Lang, D. S. Larsen, G. R. Fleming, V. Chernyak, and S. Mukamel, Phys. Rev. Lett. 79, 2702 (1997).

${ }^{3}$ K. Okumura, D. M. Jonas, and Y. Tanimura, Chem. Phys. 266, 237 (2001).

${ }^{4}$ K. Okumura and Y. Tanimura, Chem. Phys. Lett. 278, 175 (1997).

${ }^{5}$ M. Cho, K. Okumura, and Y. Tanimura, J. Chem. Phys. 108, 1326 (1998).

${ }^{6}$ N. H. Ge, M. T. Zanni, and R. M. Hochstrasser, J. Phys. Chem. A 106, 962 (2002).

${ }^{7}$ R. Venkatramani and S. Mukamel, J. Chem. Phys. 117, 11089 (2002)

${ }^{8}$ K. Okumura, A. Tokmakoff, and Y. Tanimura, J. Chem. Phys. 111, 492 (1999).

${ }^{9}$ K. A. Merchant, W. G. Noid, D. E. Thompson, R. Akiyama, R. F. Loring, and M. D. Fayer, J. Phys. Chem. B 107, 4 (2003).

${ }^{10}$ (a) C. Scheurer, A. Piryatinski, and S. Mukamel, J. Am. Chem. Soc. 123, 3114 (2001); (b) C. Scheurer and S. Mukamel, J. Chem. Phys. 116, 6803 (2002).

${ }^{11}$ W. Zhao and J. C. Wright, Phys. Rev. Lett. 84, 1411 (2000).

${ }^{12}$ M. C. Asplund, M. T. Zanni, and R. M. Hochstrasser, Proc. Natl. Acad. Sci. U.S.A. 97, 8219 (2000).

${ }^{13}$ Y. Suzuki and Y. Tanimura, J. Chem. Phys. 115, 2267 (2001).

${ }^{14}$ (a) V. Astinov, K. J. Kubarych, C. J. Milne, and R. J. D. Miller, Chem. Phys. Lett. 327, 3334 (2000); (b) K. J. Kubarych, C. L. Milne, S. Lin, V. Astinov, and J. D. Miller, J. Chem. Phys. 116, 2016 (2002).

${ }^{15}$ O. Golonzka, N. Demirdöven, M. Khalil, and A. Tokmakoff, J. Chem. Phys. 113, 9893 (2000).

${ }^{16}$ (a) L. J. Kaufman, J. Heo, L. D. Ziegler, and G. R. Fleming, Phys. Rev. Lett. 88, 207402 (2002); (b) L. J. Kaufman, D. A. Blank, and G. R. Fleming, J. Chem. Phys. 114, 2312 (2001); (c) D. A. Blank, L. J. Kaufman, and G. R. Fleming, ibid. 113, 771 (2000); (d) 111, 3105 (1999).

${ }^{17} \mathrm{~K}$. Tominaga and K. Yoshihara, J. Chin. Chem. Soc. (Taipei) 47, 631 (2000).

${ }^{18}$ (a)J. C. Kirkwood and A. C. Albrecht, J. Raman Spectrosc. 31, 107 (2000); (b) J. C. Kirkwood, A. C. Albrecht, and D. J. Ulness, J. Chem. Phys. 111, 253 (1999); (c) J. C. Kirkwood, A. C. Albrecht, D. J. Ulness, and M. J. Stimson, ibid. 111, 272 (1999).

${ }^{19}$ M. Cho, D. A. Blank, J. Sung, K. Park, S. Hahn, and G. R. Fleming, J. Chem. Phys. 112, 2082 (2000).

${ }^{20}$ K. Tominaga and K. Yoshihara, Phys. Rev. Lett. 74, 3061 (1995).

${ }^{21}$ T. Steffen and K. Duppen, Phys. Rev. Lett. 76, 1224 (1996).

${ }^{22}$ A. Tokmakoff and G. R. Fleming, J. Chem. Phys. 106, 2569 (1997).

${ }^{23}$ (a) S. Saito and I. Ohmine, J. Chem. Phys. 108, 240 (1998); (b) 106, 4889 (1997).

${ }^{24}$ T. I. C. Jansen, J. G. Snijders, and K. Duppen, J. Chem. Phys. 113, 307 (2000).

${ }^{25}$ (a) T. Keyes and J. T. Fourkas, J. Chem. Phys. 112, 287 (2000); (b) R. L. Murry, J. T. Fourkas, and T. Keyes, ibid. 109, 7913 (1998).

${ }^{26}$ (a) K. Kwac and M. Cho, J. Chem. Phys. 119, 2247 (2003); (b) 119, 2256 (2003).

${ }^{27}$ (a) A. Piryatinski, C. P. Lawrence, and J. L. Skinner, J. Chem. Phys. 118, 9664 (2003); (b) 118, 9672 (2003).

${ }^{28}$ T. I. C. Jansen, J. G. Snijders, and K. Duppen, J. Chem. Phys. 114, 10910 (2001).

${ }^{29}$ J. Kim and T. Keyes, Phys. Rev. E 65, 061102 (2002).

${ }^{30}$ S. Saito and I. Ohmine, Phys. Rev. Lett. 88, 207401 (2002).

${ }^{31}$ (a) K. Okumura and Y. Tanimura, J. Chem. Phys. 106, 1687 (1997); (b) 107, 2267 (1997).

${ }^{32}$ A. Piryatinski, V. Chernyak, and S. Mukamel, Chem. Phys. 266, 311 (2001).

${ }^{33}$ V. Khidekel, V. Chernyak, and S. Mukamel, J. Chem. Phys. 105, 8543 (1996).

${ }^{34}$ (a) S. Hahn, K. Park, and M. Cho, J. Chem. Phys. 111, 4121 (1999); (b) K. Park, M. Cho, S. Hahn, and D. Kim, ibid. 111, 4131 (1999); (c) M. Cho, ibid. 111, 4140 (1999).

${ }^{35}$ A. Ma and R. M. Stratt, J. Chem. Phys. 116, 4972 (2002).

${ }^{36}$ A. Ma and R. M. Stratt, Phys. Rev. Lett. 85, 1004 (2000).

${ }^{37}$ (a) R. A. Denny and D. R. Reichman, J. Chem. Phys. 116, 1979 (2002); (b) 116, 1987 (2002); Phys. Rev. E 63, 065101 (2001).

${ }^{38}$ (a) J. Cao, J. Wu, and S. Yang, J. Chem. Phys. 116, 3739 (2002); (b) 116, 3760 (2002).
${ }^{39}$ Y. Suzuki and Y. Tanimura, J. Chem. Phys. 119, 1 (2003).

${ }^{40}$ O. Kühn and Y. Tanimura, J. Chem. Phys. 119, 2155 (2003).

${ }^{41}$ S. Gnanakaran and R. M. Hochstrasser, J. Am. Chem. Soc. 123, 12886 (2001).

${ }^{42}$ P. Hamm, M. Lim, and R. M. Hochstrasser, J. Phys. Chem. B 102, 6123 (1998).

${ }^{43}$ (a) O. Golonzka, M. Kahlil, N. Demirdöven, and A. Tokmakoff, J. Chem. Phys. 115, 10814 (2001); (b) N. N. Demirdöven, M. Kahlil, O. Golonzka, and A. Tokmakoff, J. Phys. Chem. A 105, 8025 (2001); (c) O. Golonzka, M. Kahlil, N. Demirdöven, and A. Tokmakoff, Phys. Rev. Lett. 86, 2154 (2001); (d) N. Demirdöven, M. Kahlil, and A. Tokmakoff, ibid. 89, 237401 (2002).

${ }^{44}$ K. Tominaga and H. Maekawa, Bull. Chem. Soc. Jpn. 74, 279 (2001).

${ }^{45}$ (a) A. M. Moran, S. M. Park, J. Dreyer, and S. Mukamel, J. Chem. Phys. 118, 3651 (2003); (b) A. M. Moran, J. Dreyer, and S. Mukamel, ibid. 118, 1347 (2003).

${ }^{46}$ (a) S. Woutersen and P. Hamm, J. Phys. Chem. B 104, 11316 (2000); 115, 7737 (2001).

${ }^{47}$ S. Mukamel, Principles of Nonlinear Optical Spectroscopy (Oxford University Press, New York, 1995).

${ }^{48} \mathrm{M}$. Cho, in Advances in Multi-photon Process and Spectroscopy, edited by S. H. Lin, A. A. Villaeys, and Y. Fujimura (World Scientific, Singapore, 1999), Vol. 12, p. 229.

${ }^{49}$ S. Mukamel, Annu. Rev. Phys. Chem. 51, 691 (2000).

${ }^{50}$ J. T. Fourkas, Adv. Chem. Phys. 117, 235 (2001).

${ }^{51}$ J. C. Wright, Int. Rev. Phys. Chem. 21, 185 (2002).

${ }^{52}$ (a) M. T. Zanni, S. Gnanakaran, J. Stenger, and R. M. Hochstrasser, J. Phys. Chem. B 105, 6520 (2001); (b) I. V. Rubtsov and R. M. Hochstrasser, ibid. 105, 6520 (2001).

${ }^{53}$ S. Woutersen, R. Pfister, P. Hamm, Y. Mu, D. S. Kosov, and G. Stock, J. Chem. Phys. 117, 6833 (2002).

${ }^{54}$ N. H. Ge and R. M. Hochstrasser, Phys. Chem. Comm. 3, 1 (2002).

${ }^{55}$ M. Cho, Phys. Chem. Comm. 7, 1 (2002).

${ }^{56}$ U. Weiss, Quantum Dissipative Systems, 2nd ed. (World Scientific, Singapore, 1999).

${ }^{57}$ H. Grabert, P. Schramm, and G. L. Ingold, Phys. Rep. 168, 115 (1988).

${ }^{58}$ P. Hänggi, P. Talkner, and M. Borkovec, Rev. Mod. Phys. 62, 251 (1990).

${ }^{59}$ A. O. Caldeira and A. J. Leggett, Physica A 121, 587 (1993).

${ }^{60}$ (a) Y. J. Yan and S. Mukamel, J. Chem. Phys. 89, 5160 (1988); (b) 94, 179 (1991).

${ }^{61}$ T. Kato and Y. Tanimura, J. Chem. Phys. 6221, 117 (2002).

${ }^{62}$ (a) D. W. Oxtoby, Adv. Chem. Phys. 40, 1 (1979); (b) 47, 487 (1981).

${ }^{63}$ J. S. Bader and B. J. Berne, J. Chem. Phys. 100, 8359 (1994).

${ }^{64}$ W. T. Pollard and R. A. Friesner, J. Chem. Phys. 100, 5054 (1994).

${ }^{65}$ K. Okumura and Y. Tanimura, Phys. Rev. E 56, 2747 (1997).

${ }^{66}$ T. Steffen and Y. Tanimura, J. Phys. Soc. Jpn. 69, 3115 (2000).

${ }^{67}$ Y. Tanimura and T. Steffen, J. Phys. Soc. Jpn. 69, 4095 (2000).

${ }^{68} \mathrm{Y}$. Tanimura, in Two-Dimensional Correlation Spectroscopy, edited by Y. Ozaki and I. Noda (Wiley, New York, 2002), p. 144.

${ }^{69}$ R. Kubo, M. Toda, and N. Hashitsume, Statistical Mechanics (Springer, New York, 1985).

${ }^{70}$ (a) Y. Tanimura and R. Kubo, J. Phys. Soc. Jpn. 58, 1199 (1989); (b) 68, 101 (1989).

${ }^{71}$ (a) Y. Tanimura and P. G. Wolynes, Phys. Rev. A 43, 4131 (1991); 96, 8485 (1992)

${ }^{72}$ Y. Tanimura, Chem. Phys. 233, 217 (1998).

${ }^{73}$ (a) R. P. Feynman and A. R. Hibbs, Quantum Mechanics and Path Integrals (McGraw-Hill, New York, 1965); (b) R. P. Feynman and F. L. Vernon, Ann. Phys. (N.Y.) 24, 118 (1963).

${ }^{74}$ K. Blum, Density Matrix Theory and Applications (Plenum, New York, 1981).

${ }^{75}$ E. Wigner, Phys. Rev. 40, 749 (1932).

${ }^{76}$ R. Kubo, J. Phys. Soc. Jpn. 19, 2127 (1964).

${ }^{77}$ W. R. Frensley, Rev. Mod. Phys. 62, 745 (1990).

${ }^{78}$ (a) Y. Tanimura and Y. Maruyama, J. Chem. Phys. 107, 1779 (1997); (b) Y. Maruyama and Y. Tanimura, Chem. Phys. Lett. 292, 28 (1998).

${ }^{79}$ T. Steffen, J. T. Fourkas, and K. Duppen, J. Chem. Phys. 105, 7364 (1996).

${ }^{80}$ J. T. Fourkas, H. Kawashima, and K. A. Nelson, J. Chem. Phys. 103, 4393 (1995).

${ }^{81} \mathrm{~K}$. Okumura and Y. Tanimura, J. Chem. Phys. (to be published).

${ }^{82}$ Y. Ohtsuki and Y. Fujimura, J. Chem. Phys. 91, 3903 (1989).

${ }^{83}$ T. Kato, M. Hayashi, A. A. Villaeys, and S. H. Lin, Phys. Rev. A 56, 980 (1997). 
${ }^{84}$ T. Steffen and K. Duppen, Chem. Phys. 233, 267 (1998).

${ }^{85}$ (a) M. Shiga and S. Okazaki, J. Chem. Phys. 109, 3542 (1998); (b) 111, 5390 (1999).

${ }^{86}$ T. Kato and Y. Tanimura, Chem. Phys. Lett. 341, 329 (2001).

${ }^{87}$ Y. J. Yan, F. Shuang, R. Xu, J. Cheng, X. Q. Li, C. Yang, and H. Zhang, J. Chem. Phys. 113, 2068 (2000).

${ }^{88}$ J. Sung and M. Cho, J. Chem. Phys. 113, 7072 (2000).

${ }^{89}$ J. Sung, R. J. Silbey, and M. Cho, J. Chem. Phys. 115, 1422 (2001).

${ }^{90}$ M. Cho, J. Chem. Phys. 115, 4424 (2001).

${ }^{91}$ D. V. Bout, L. J. Muller, and M. Berg, Phys. Rev. Lett. 67, 3700 (1991).
${ }^{92}$ D. Zimdars, A. Tokmakoff, S. Chen, S. R. Greenfield, M. D. Fayer, T. I. Smith, and H. A. Schwettman, Phys. Rev. Lett. 70, 2718 (1993).

${ }^{93}$ K. A. Merchant, D. E. Thompson, and M. D. Fayer, Phys. Rev. Lett. 86, 3899 (2001).

${ }^{94}$ (a) V. Khidekel and S. Mukamel, Chem. Phys. Lett. 204, 304 (1995); (b) 263, 350 (1996)

${ }^{95}$ R. Inaba, K. Tominaga, M. Tasumi, K. A. Nelson, and K. Yoshihara, Chem. Phys. Lett. 211, 183 (1993).

${ }^{96}$ (a) R. B. Williams and R. F. Loring, J. Chem. Phys. 113, 1932 (2000); (b) 110, 10899 (1999). 